\title{
Approche probabiliste des efforts extrêmes sur une structure sensible à la période des vagues
}

\author{
Probalistic approach of extreme stresses \\ on structures sensitive to wave periods.
}

\author{
Geneviève Lebas
}

Elf-Aquitaine

Le calcul probabiliste des efforts extrêmes sur une structure est décrit, en prenant en compte l'incertitude sur les fonctions de transfert (qui permettent de calculer l'effort correspondant à une vague de hauteur et période données), et les distributions conjointes des hauteurs et des périodes des vagues sur le site d'implantation.

Globalement, une structure doit supporter un moment de renversement et une force horizontale. L'étude des efforts engendrés par les couples hauteur-période centennaux a montré que force et moment n'étaient pas entièrement corrélés et qu'il n'y avait donc pas lieu d'appliquer, lors du calcul, simultanément leurs valeurs extrêmes.

The probalistic calculation of extreme stresses is described whilst taking into account the uncertainty concerning the transfer functions (which allow for the stress corresponding to a high wave and data periods), and the joint distributions of heights and periods of wave disturbances on the installation site.

Overall, a structure must bear a moment of change and a horizontal force. A study of the stresses generated by the centennial height-period pairings has shown that force and moment were not entirely correlated and that there was therefore no need to apply simultaneously, at the time of calculation, their extreme values.

\section{Introduction}

L'objectif de cette étude est d'estimer les efforts extrêmes à prendre en compte sur une structure en Mer du Nord.

De simples variations de paramètres mal connus pouvant donner des différences importantes sur le résultat, l'étude de la distribution des efforts était nécessaire.

Les calculs prennent en compte les incertitudes sur ces paramètres mal connus, ainsi que les distributions du chargement, c'est-à-dire la houle.

L'analyse statistique des mesures de vagues réalisées sur le site nous a permis de calculer la distribution des vagues extrêmes sur différentes périodes de retour.

Les calculs d'efforts sont déduits d'une fonction de transfert dont l'incertitude est décrite dans une étude. 


\section{Conclusion}

Avec les mesures réalisées sur le champ, l'étude de la distribution conjointe, hauteur et période des vagues a pu être réalisée. Pour toutes les vagues, d'une même probabilité d'apparition correspondant à une période de retour, la force horizontale et le moment de renversement ont été calculés.

La force maximale est de 72500 tonnes et est obtenue pour une vague de hauteur 28.8 mètres et de période 16.2 secondes, alors que la vague de hauteur extrême $(\mathrm{H}=29.6 \mathrm{~m}$ et $\mathrm{T}=14.7 \mathrm{~s})$ induit une force de 67300 tonnes.

Le moment de renversement maximal $\left(3.510^{6}\right.$ ton- nes-mètres) est obtenu approximativement pour la vague de hauteur extrême.

Les vagues extrêmes donnant les efforts maximaux ne sont pas exactement, les mêmes, elles ne surviendront pas ensemble, bien que le moment et la force sont dans ce cas bien correlés.

Prenant en compte une incertitude sur la fonction de transfert et ne considérant plus les vagues extrêmes les plus probables, mais leur probabilité d'apparition, nous pouvons déduire la distribution des efforts extrêmes.

Les décisions qui seront prises par la suite seront donc associées à un risque de dépassement.

\section{Fonction de transfert}

Les erreurs dues à la formule de Morison sont assez mal connues, et donc la fonction de transfert (fonction qui permet de passer d'une vague donnée à un effort) $R(T)$ ne peut être considérée certaine. Cependant, on peut estimer que $R(T)$ suit une loi normale avec un coefficient de variation de $12 \%$. Ainsi pour une vague donnée de période $T$, on calcule la moyenne et la variance de $R(T)$.

On a donc la distribution conditionnelle de $R$ pour $T$

$$
f(R \mid T)
$$

les figures 1 et 2, ci-dessous, montrent les moyennes et les limites à $95 \%$ des fonctions de transfert.

\section{Distribution des vagues extrêmes}

L'analyse statistique des mesures effectuées sur le champ donne les distributions des hauteurs de vagues extrêmes $\mathrm{fe}(\mathrm{H})$. La figure 3 montre la densité de probabilité de la hauteur extrême sur 100 ans.

Cette densité de probabilité peut être décrite, en premier, par la valeur extrême la plus probable (celle qui a la plus grande probabilité d'occurence sur cette période). Il est important de noter que la probabilité de rencontrer une vague extrême supérieure à celle-ci est de $63 \%$ alors que la probabilité d'en rencontrer une qui soit inférieure est de $37 \%$.

Comme le montrent les courbes de transfert, il est nécessaire d'associer une période à la hauteur. Les mesures de vagues (figure 4) montrent une dispersion

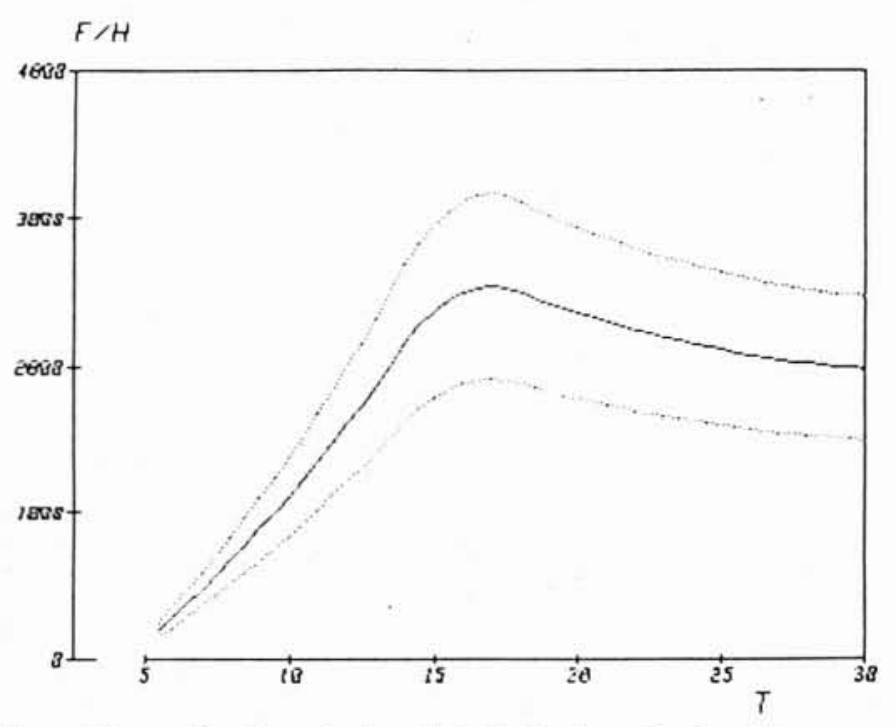

Figure 1. - Fonction de transfert de la force horizontale : moyenne et limite à $95 \%$ fonction de la période $\mathrm{T}$.

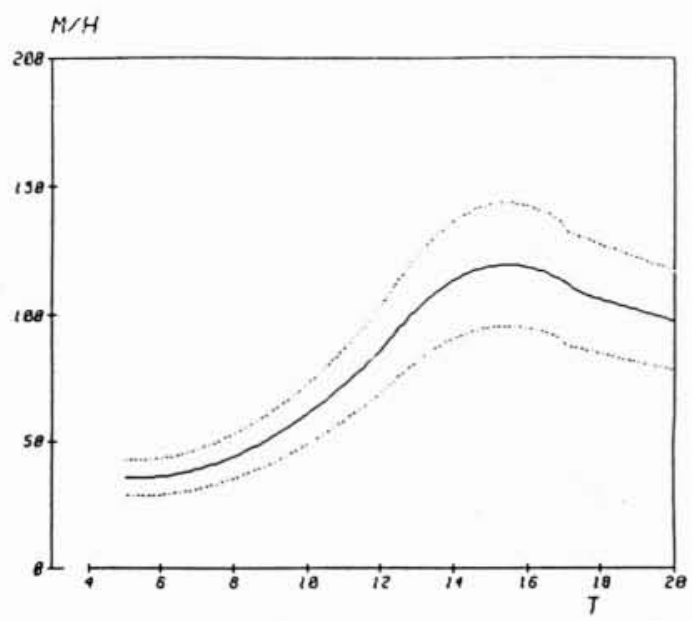

Figure 2. - Fonction de transfert du moment de renversement : moyenne et limite à $95 \%$ fonction de la période $\mathrm{T}$. 
importante des périodes associées à une hauteur. Ces mesures nous permettent d'estimer la distribution des périodes pour une hauteur maximum d'un état de mer. Tous les histogrammes sont correctement ajustés par une loi de Wald (Inverse Gaussienne). La figure 5 montre un exemple d'ajustement et le compare avec la loi normale. Cette distribution est décrite par la moyenne $m$, et un paramètre de dispersion $\lambda$, qui peuvent s'exprimer en fonction de la hauteur comme le montre les figures 6 et 7. Ainsi, on estime la densité de probabilité d'une période pour une hauteur de vague donnée $f(T \mid H)$.

Pour une hauteur de vague, on peut donc estimer la période associée la plus probable et pour une période de retour, toutes les vagues extrêmes (hauteur et période) qui ont la même probabilité d'apparition.

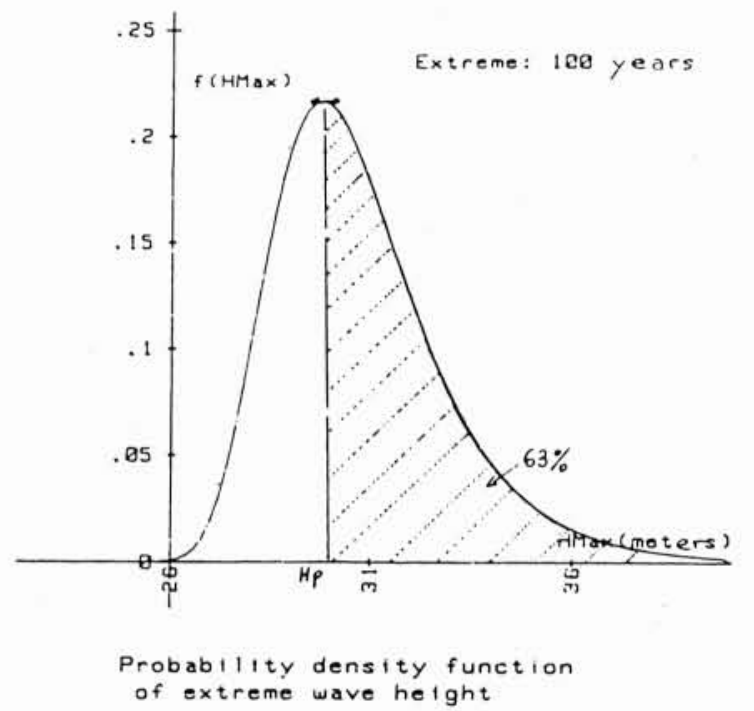

Figure 3. - Distribution de la hauteur de vague extrême pour une période de retour de 100 ans.

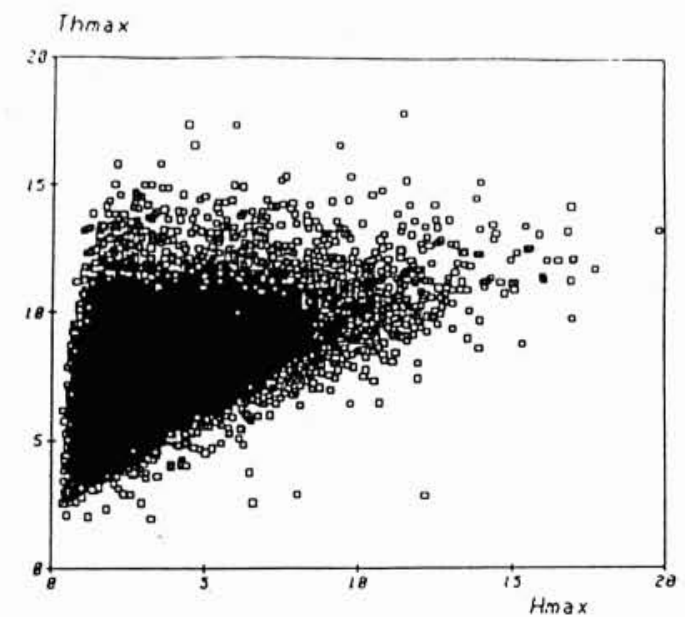

Figure 4. - Diagramme de dispersion : hauteur de vague maxi de l'état de mer et leur période.
LOI MORMale

neyenne: $: 6.516 \cdot 00$
varaince: $: 3.665 \cdot 00$ Chi-deux: $196+01$

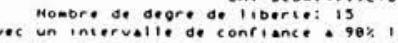

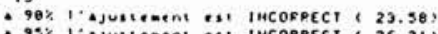

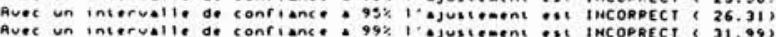

2 LOI DE MALD

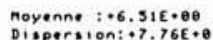

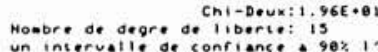

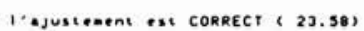

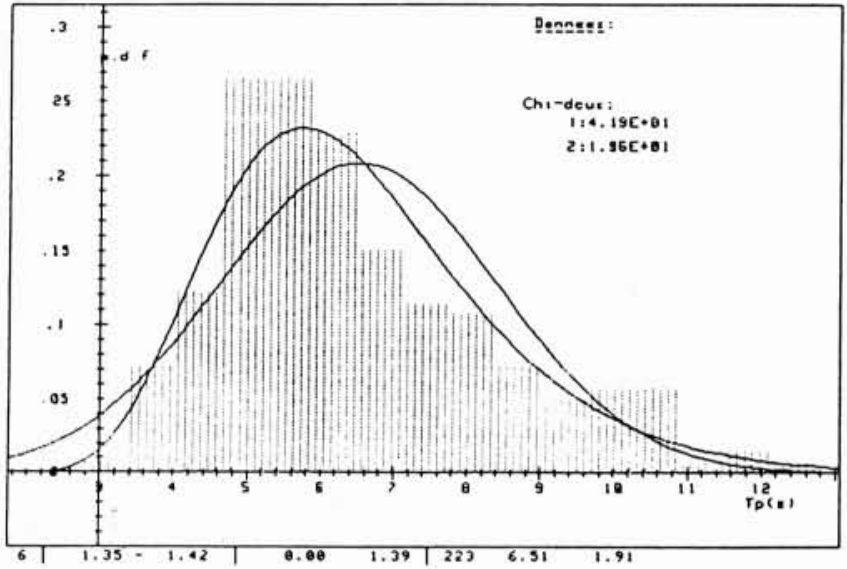

Figure 5. - Histogramme des périodes de vagues pour une hauteur donnée : ajustement par une loi normale et une loi de Wald.

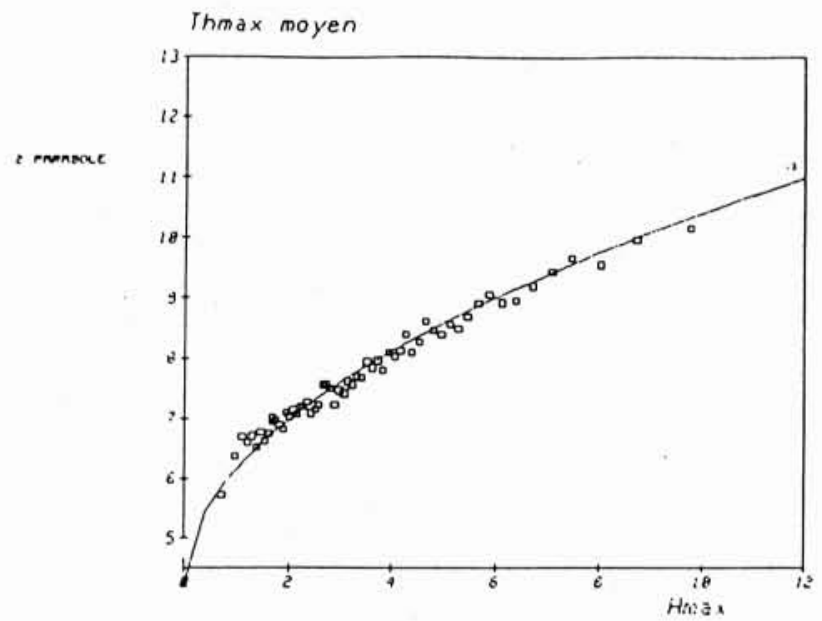

Figure 6. - Moyenne des périodes en fonction de la hauteur.

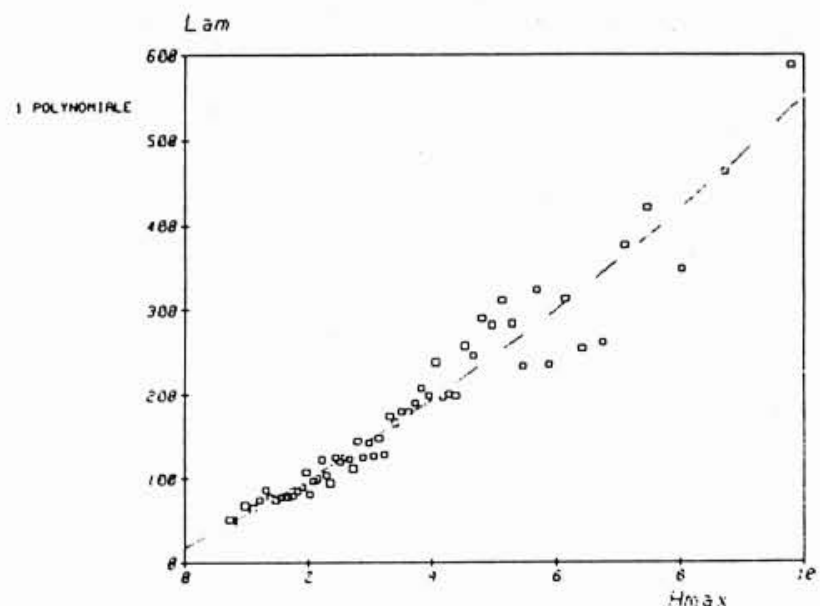

Figure 7. - Paramètre de dispersion en fonction de la hauteur. 


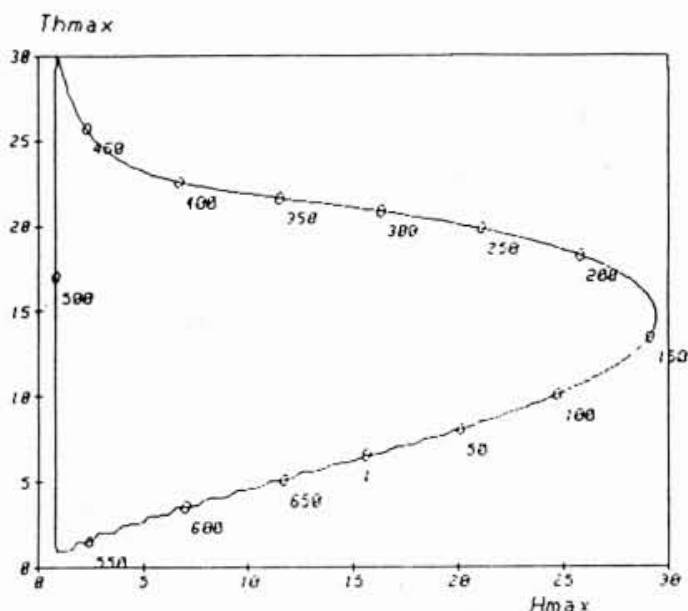

Figure 8. - Vagues de même probabilité.

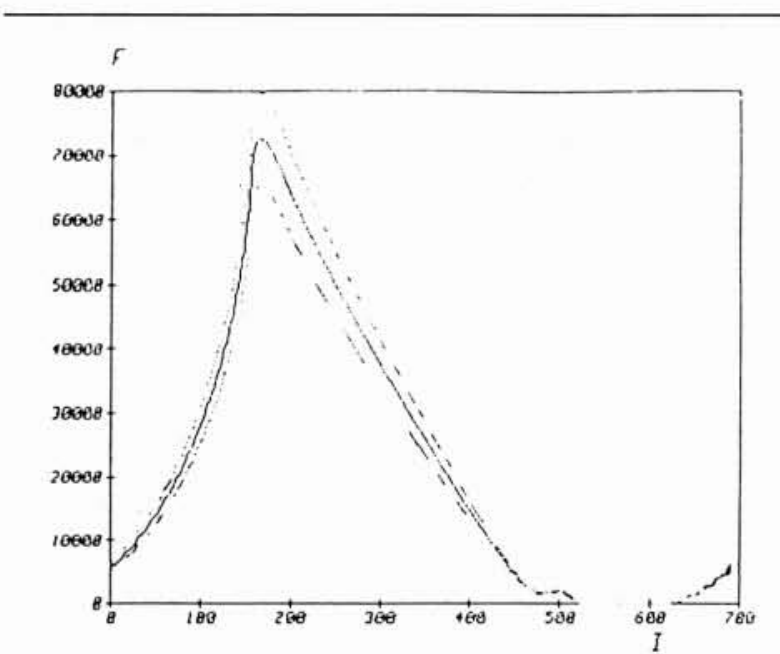

Figure 9. - Force horizontale en fonction des vagues extrêmes indexées de 1 à 700 .

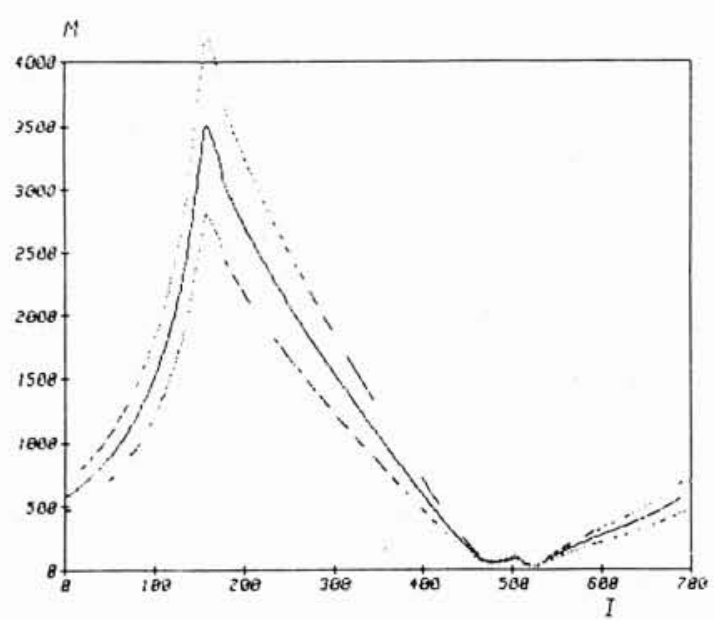

Figure 10. - Moment de renversement en fonction des vagues extrêmes indexées de 1 à 700 .

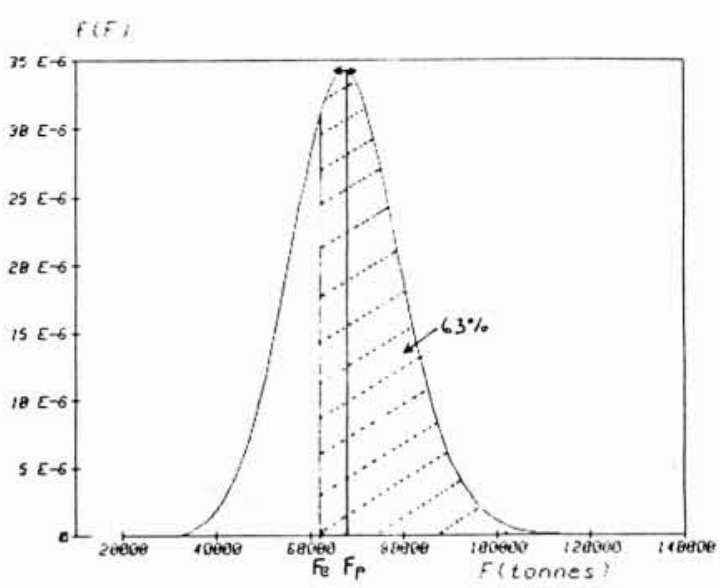

Figure 11. - Densité de probabilité de la force horizontale extrême sur une période de 50 ans.

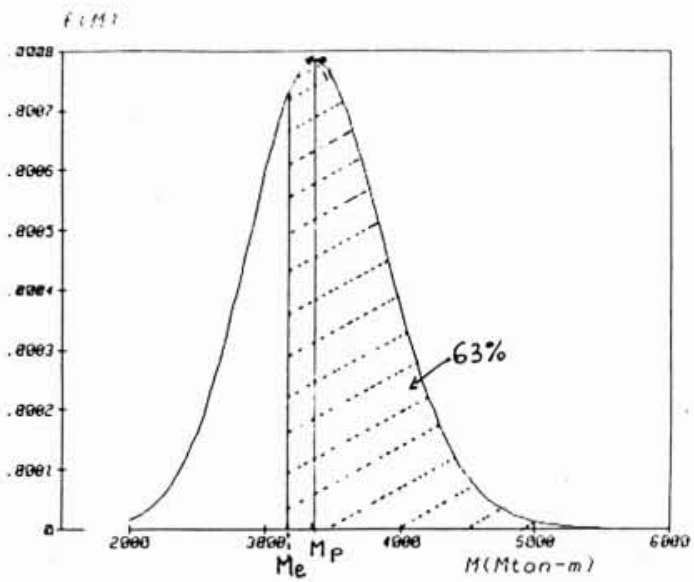

Figure 12. - Densité de probabilité du moment de renversement extrême sur une période de 50 ans. 
Pour une période de retour de 100 ans, ces couples sont représentés par la figure 8 . Par exemple, ces vagues ont la même probabilité d'apparition sur 100 ans :

1. $H=29.5 \mathrm{~m}$ et $T=14.7 \mathrm{~s}$

2. $H=20$ m et $T=20 \mathrm{~s}$

3. $H=20$ m et $T=7.6 \mathrm{~s}$

Laquelle est la plus pénalisante pour la structure ?

\section{Efforts}

\section{Corrélations}

Pour toutes les vagues représentées par la figure 8 , et indexées de 1 à 700 , nous avons calculé la force horizontale (figure 9) et le moment de renversement (figure 10).

Dans cette application, on peut en première approximation considérer que le moment maximum et la force maximum, sont donnés par la même vague. Moment et force sont très bien corrélés car les fonctions de transfert ont la même forme et le maximum correspond à la période la plus probable. On remarque que prendre en compte les deux maximum est une borne supérieure dans le calcul de la structure, et que ces maximum ne sont pas donnés forcément par la vague de hauteur extrême $(H=$ 29.4 mètres) la force est maxi $(72500 \mathrm{t}$ ) pour $H=28.8 \mathrm{~m}$ et $T=16.2 \mathrm{~s}$ alors qu'elle vaut 67300 t pour la vague de hauteur extrême.

\section{Distribution}

Ainsi on connait la vague la plus pénalisante pour la structure. Toutefois, les efforts engendrés par cette vague présentent une dispersion due aux distributions de hauteur et période, ainsi qu'à l'incertitude de la fonction de transfert.

Pour une période de retour, on connait la distribution de la hauteur extrême $f e(H)$, pour une hauteur $H$, on estime la distribution des périodes associées $f_{T}(T \mid H)$ et finalement pour une période on en déduit une distribution de la fonction de transfert $f_{R}(R \mid T)$.

La distribution de la fonction de transfert, pour une vague donnée, est donc :

$$
\begin{gathered}
f_{R}(R \mid H)=\int_{0}^{\infty} f_{R}(R \mid T) f_{T}(T \mid H) d H \\
f_{R}(R)=\int_{0}^{\infty} f_{R}(R \mid H) f e(H) d H
\end{gathered}
$$

et la distribution de la force $F$, pour une période de retour est :

$$
f_{F}(F)=\int_{0}^{\infty} \frac{1}{H} f_{R}\left(\frac{F}{H}\right) f e(H) d H
$$

On effectue le calcul analogue pour le moment de renversement.
Il faut remarquer que la densité de probabilité ainsi obtenue est une loi normale (la fonction de transfert étant une loi normale). La figure 11 représente la densité de probabilité de la force horizontale pour une période de retour de 50 ans.

La dispersion est importante et la difficulté est de choisir une valeur, ou un risque de dépassement.

Chacun peut avoir son critère de choix. En bonne logique avec la distribution des hauteurs de vagues extrêmes, on peut suggérer la valeur la plus probable $F_{p}$ ou celle qui a une probabilité de $63 \%$ d'être dépassée, $\mathrm{Fe}$.

La figure 12 représente la même distribution pour le moment de renversement.

\section{Bibliographie}

[1] Probability, Statistics and Decision for Civil Engineer. J.R. Benjamin et A. Cornell.

[2] Continuous univariate distribution 1. 2. Norman L. Johnson et S. Kotz. Wiley Series in probability and Mathematical Statistic. 
M. M le Président Banal: Qui désire poser une question à $\mathrm{M}^{\mathrm{lt}}$ LEBAS?

M. HAMM : J'ai juste été choqué par un petit détail pratique. Lorsqu'on cite une amplitude de 20 mètres associée à une période de 7 secondes, est-ce physiquement réel ?

$M^{\text {Ite }}$ LEBAS : On limite des distributions estimées par la courbe de déferlement des vagues donnée par MM. SUSBIELLES et BRATUT.

M. BOUlon: Pour que ce soit clair, quand on parle d'amplitude, c'est l'amplitude crête à creux.

$M^{\text {Ite }}$ LEBAS: Oui, il s'agit de l'amplitude de la vague crête à creux déterminée par la méthode up zéro crossing. fert?

M. LaURAS: Comment obtenez-vous des fonctions de trans-

$M^{\text {the }}$ LEBAS: C'est la fonction de transfert prise par le conducteur de la structure. De plus, des essais réalisés en bassin ont montré une dispersion qui pouvait être estimée par un coefficient de variation de $12 \%$. On a appliqué ce coefficient à la fonction de transfert Skjelbreia, établie en 1975.

M. DARRAS : Je voudrais faire un commentaire sur l'événement extrême.

Effectivement on peut se poser la question qui vient d'être soulevée, à savoir si les expressions de distribution statistique correspondent à des phénomènes physiques. C'est quelque chose qu'il faut garder en tête quand on fait des extrapolations et il faut certainement se poser la question du phénomène générateur.

Avoir 20 mètres avec 7 secondes, cela doit pouvoir exister, ne serait-ce que dans des sites croisés où il se peut très bien que des vagues se croisent à l'endroit de la structure.

Mais j'avais en fait une question concernant les méthodes d'extrapolation à deux paramètres puisque vous êtes obligée pour déterminer des couples hauteur-période correspondant à une durée de retour de cent ans, de faire une extrapolation à deux paramètres. Cette extrapolation à deux paramètres nécessite d'avoir un critère de dépassement et j'aimerais que vous essayiez d'expliquer comment vous avez fait votre choix en fonction de la structure que vous avez. Pour notre part nous avons un problème similaire avec le couple hauteur-durée, rencontré dans les problèmes de digues.

$M^{\text {Ite }}$ LEBAS: En ce qui concerne la première partie, on commence à avoir un certain nombre de données et à partir de là, on peut calculer une distribution conjointe hauteur-période. On s'aperçoit que les courbes d'isoprobabilité correspondant à des périodes de retour à 1,2 ou 5 ans (puisque l'on a 5 ans de données) entoure bien le nuage de points. On peut alors considérer avec des moyens statistiques en plus, que la distribution hauteurpériode est bonne. Il y a de nombreux moyens de vérification.
Ceci pour la première partie de votre intervention.

En ce qui concerne la deuxième partie : l'extrapolation, je n'a pas calculé une distribution conjointe des vagues extrêmes, hauteur-période. J'ai simplement pris la courbe des couples extrêmes les plus probables sur cent ans.

On a l'habitude d'extrapoler suivant une direction et de calculer la distribution de la hauteur extrême, qui se traduit par la valeur la plus probable et un risque de dépassement. Pour l'instant, nous n'avons pas fait d'extrapolation à deux dimensions. Nous avons simplement calculé les courbes des vagues hauteurpériode ayant même probabilité d'apparition sur 100 ans.

M. COUPRIE : Au point de vue interaction sur la structure, vous avez reconstitué le moment, la force horizontale, il reste la force verticale car le caisson se remplit et se vidange d'eau; pour l'interaction avec le sol, il faut savoir quelle est la force verticale qui n'est pas constante. Avez-vous, dans votre étude, eu l'occasion d'aborder cette troisième grandeur qui n'est pas traduite dans votre texte?

$M^{H_{t}}$ LEBAS: On n'a pas estimé la distribution de la force verticale. Le problème posé était celui d'une structure un peu endommagée, dont le calcul déterministe de la force horizontale et du moment de renversement ne satisfaisaient pas les règlementations.

II s'agissait donc de prendre en compte les incertitudes des paramètres mal connus et d'estimer la dispersion de cette force et de ce moment, pour estimer le risque de dépassement.

M. COUPRIE : $\mathrm{Si}$ vous voulez traiter les problèmes de plateforme dans leur totalité il faut connaître le troisième élément qui est la force verticale. Ce n'est pas parce que vous améliorez votre connaissance des moments et des forces horizontales que vous réglez la question. Il faut traiter les trois éléments avec des données équivalentes et la photo que vous venez de présenter, très intéressante, montrait que le caisson se vidangeait. Le bac était rempli aux $2 / 3$ de la hauteur de la houle. Vous vous imaginez que toute cette eau pèse au fond; actuellement cela fait une vingtaine de mètres d'eau; soit une force importante comparée aux contraintes totales; pour parler comme les géotechniciens, il y a deux bars qui fluctuent, alors que la contrainte totale due au moment est de 3,4 bars - c'est un ordre de grandeur - est très considérable.

$M^{\text {te }}$ LEBAS : J'ai dit que le cas de force verticale n'avait pas été calculé de façon probabiliste, mais bien sûr, la force verticale ainsi que l'interaction avec le sol ont été pris en compte de façon déterministe. Le calcul probabiliste de la force horizontale et du moment de renversement ont été un premier pas dans l'approche probabiliste et ont permis de montrer que le phénomène est plus complexe et que pour prendre toutes les incertitudes en compte, il reste encore beaucoup de choses à faire. 